\title{
MODELAGEM E CONTROLE DISCRETO PARA O RETIFICADOR PFC BOOST TRÊS NÍVEIS
}

\author{
J.E. Baggio, H.L. Hey, H.A. Gründling, H. Pinheiro, J.R. Pinheiro \\ Universidade Federal de Santa Maria \\ CT / NUPEDEE / GEPOC \\ 97105-900 - Santa Maria - RS - Brasil \\ E-mail: josebaggio@ieee.org, renes@ctlab.ufsm.br
}

\begin{abstract}
Resumo - Este artigo apresenta um controlador discreto para o retificador boost três níveis com fator de potência corrigido. Modelos discretos lineares de primeira ordem para ambas as malhas de tensão e corrente são apresentadas. Controladores servos com realimentação de estados são utilizados, sendo projetados utilizando-se um regulador linear quadrático discreto. Além disto, um observador de estados preditivo é utilizado para compensar o tempo de atraso associado à implementação em processadores digitais. É apresentada uma descrição detalhada da lógica de comando que garante a divisão simétrica de tensão do barramento cc. Resultados experimentais de um retificador de $1.5 \mathrm{~kW}$ são apresentados para validar a análise desenvolvida bem como para demonstrar a boa performance do sistema.
\end{abstract}

\begin{abstract}
This paper presents a discrete control for the three-level boost power factor corrected rectifier. Linearized discrete models for both the current loop and for the voltage loop are shown. Then, servo controllers with state feedback are developed and designed using the discrete linear quadratic regulator approach. In addition, a predictive state observer is employed to compensate the delay time associated to the discrete implementation. Furthermore, a detailed description of the logic command that assures half de bus sharing is described. Finally, experimental results from a $1.5 \mathrm{~kW}$ TLB-PFC rectifier are presented to validate the analysis carried out as well as to demonstrate the good performance of the system.
\end{abstract}

\section{INTRODUÇÃO}

O retificador boost três níveis com fator de potência corrigido (Three-level boost power factor corrected TLBPFC) está sendo cada vez mais utilizado devido às seguintes vantagens: (i) os interruptores e diodos são projetados para suportar somente a metade da tensão do barramento cc; (ii) o volume do indutor do TLB é um quarto do volume do indutor do boost convencional $[1,2]$; (iii) é possível a regulação da tensão do ponto central do barramento cc [2]. Como resultado, este conversor é atrativo para aplicações de alta tensão no barramento cc e alta potência.

Circuitos integrados dedicados para correção de fator de potência têm sido amplamente utilizados pela industria. Nestes casos, os controladores são geralmente projetados baseados em modelos de pequenos sinais e/ou modelos médios no domínio de tempo contínuo [2,3,4,5]. Por outro lado, controladores digitais estão se tornando cada vez mais baratos, tornando-os fortes candidatos para a substituição dos circuitos analógicos convencionais [6,7,8]. Isto ocorre principalmente devido à facilidade da implementação de técnicas de controle avançadas em controladores digitais sem que a complexidade dos circuitos do controlador aumente.

Existem basicamente dois modos de se projetar um controlador digital: (i) o controlador é projetado baseado em um modelo contínuo e posteriormente é discretizado para a implementação digital $[2,13,14]$; (ii) o modelo do sistema no domínio de tempo contínuo é discretizado e então um controlador digital é projetado no domínio de tempo discreto. O primeiro método geralmente é preferível uma vez que as técnicas de controle no domínio de tempo contínuo são bem conhecidas. Entretanto, a performance do sistema depende do método adotado para a discretização do controlador $[8,9,14]$. Por outro lado, o segundo método é preferível quando um modelo discreto acurado da planta é obtido, permitindo-se utilizar todas as vantagens da capacidade de processamento dos controladores digitais.

Neste artigo, o segundo método foi adotado, e procedimentos sistemáticos de projeto são apresentados utilizando representação por variáveis de estado para a malha interna de corrente e para a malha externa de tensão.

A lógica de chaveamento que garante a divisão simétrica de tensão do barramento cc para o TLB-PFC proposta em [2] é utilizada. Desta forma, um modelo não linear considerando a lógica de chaveamento utilizada é obtido. $\mathrm{O}$ modelo não linear é então linearizado, resultando em um modelo discreto simples de primeira ordem linear invariante no tempo (LTI), que representa o comportamento discreto da corrente no indutor do retificador TLB-PFC. Da mesma forma, um modelo discreto de primeira ordem invariante no tempo é utilizado para o projeto do controlador da tensão do barramento cc.

Controladores servos com realimentação de estados foi escolhido para ambas a malha interna de corrente e a malha externa de tensão. Para se obter um procedimento sistemático para o projeto dos controladores, o regulador linear quadrático discreto (discrete linear quadratic regulator DLQR) para regime permanente foi escolhido como método para obtenção dos ganhos dos controladores. Além disto, um observador de estados preditivo é empregado para compensar o tempo de atraso associado à implementação discreta da lei de controle da malha interna de corrente.

Resultados experimentais de um protótipo de $1,5 \mathrm{~kW}$ são apresentados para demonstrar a performance do sistema proposto, bem como o comportamento do retificador TLBPFC com o controlador discreto proposto. 


\section{MODELAGEM DO CONVERSOR}

A Fig. 1 apresenta o diagrama de blocos do controlador digital para o retificador TLB-PFC. A estrutura adotada para o controlador segue a bem conhecida e utilizada técnica onde a malha externa de tensão opera em baixa freqüência e regula a tensão do barramento cc, enquanto que uma malha rápida interna de corrente regula a corrente no indutor boost, com alto fator de potência $[2,3,4,6,11]$. A corrente de referência $i_{r e f}$ para a malha interna de corrente é obtida multiplicando-se a tensão de entrada retificada medida pela saída do controlador do barramento cc $\left(u_{c c}\right)$. O sinal de controle $u$ gerado pela malha de corrente é utilizado na lógica de comando que define qual interruptor entra em condução ou é bloqueado.
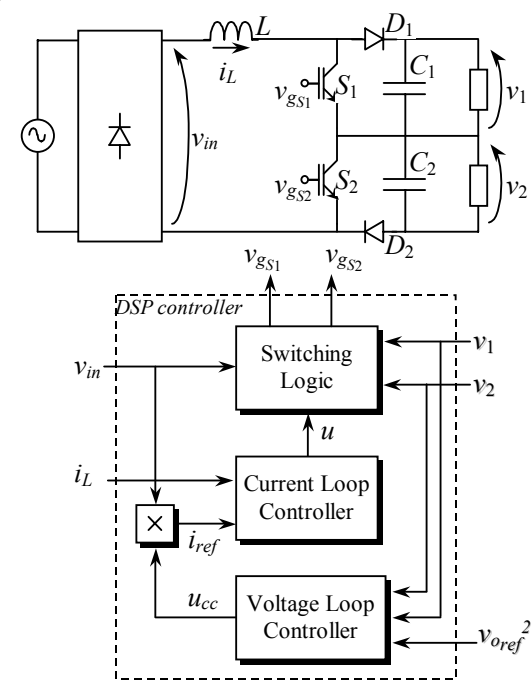

Fig. 1 - Retificador TLB-PFC com controle digital.

Nesta seção são apresentados os modelos discretos propostos para o projeto dos controladores.

\subsection{Lógica de Chaveamento para a Divisão Simétrica da Tensão do Barramento cc}

$\mathrm{O}$ retificador TLB-PFC operando em modo de condução contínua (CCM) pode apresentar quatro etapas de operação, conforme apresentado na Fig. 2.

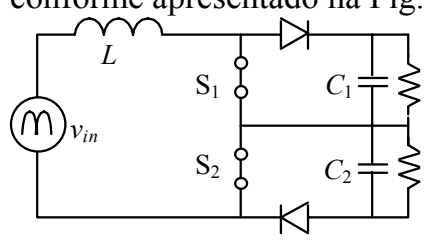

(a) Etapa 1

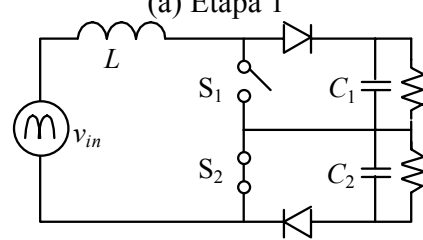

(c) Etapa 3

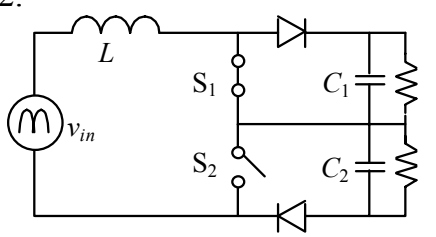

(b) Etapa 2

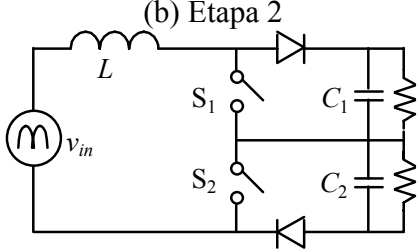

(d) Etapa 4
Fig. 2 - Etapas de operação do retificador TLB-PFC em CCM.

A lógica de chaveamento que permite a divisão simétrica de tensão do barramento cc mesmo para cargas desbalanceadas foi apresentada em [2]. Esta técnica utiliza a energia armazenada no indutor para equalizar a tensão nos capacitores $C_{1}$ e $C_{2}$. Desta forma, nos estágios 2 e 3, o interruptor que está em condução ou está bloqueado é definido pela comparação entre as tensões nos capacitores. $\mathrm{O}$ uso desta técnica é indicado quando cargas tais como conversores dc-dc multíniveis consomem potência desbalanceada das duas tensões de saída do retificador TLBPFC. A lógica de chaveamento utilizada é apresentada na Tabela 1.

\section{TABELA I}

\section{Lógica de Chaveamento}

\begin{tabular}{c|c|c|c|c|}
\cline { 2 - 5 } & \multicolumn{2}{c|}{ Região $1\left(v_{\text {in }}<v_{1}\right)$} & \multicolumn{2}{c|}{ Região $2\left(v_{\text {in }}>v_{1}\right)$} \\
\cline { 2 - 5 } & $v_{1}<v_{2}$ & $v_{1}>v_{2}$ & $v_{1}<v_{2}$ & $v_{1}>v_{2}$ \\
\hline $\mathrm{S}_{1}$ & PWM & ON & OFF & PWM \\
\hline $\mathrm{S}_{2}$ & ON & PWM & PWM & OFF \\
\hline
\end{tabular}

A lógica de comando apresentada na TABELA I, foi implementada por software, e os sinais de comando ( $P W M$, $O N, O F F)$ foram obtidos utilizado os geradores PWM internos do DSP. Para a geração do sinal $O N$, carrega-se o comparador interno do gerador PWM com o valor máximo, enquanto que para a geração do sinal OFF, carrega-se o comparador com zero. O sinal $P W M$ é obtido carregando o comparador interno do gerador PWM com o valor obtido pela lei de controle, $u$.

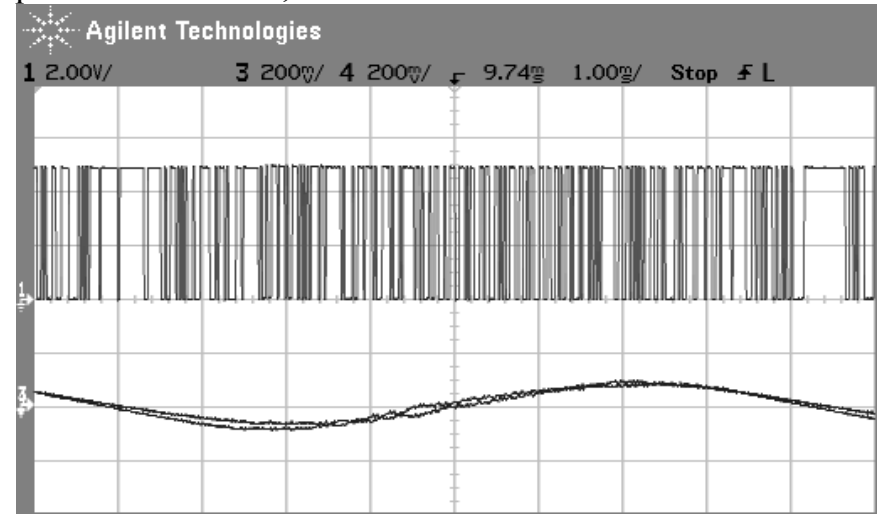

Fig. 3 - Divisão simétrica de tensão do barramento cc.

A Fig. 3 demonstra a divisão simétrica de tensão no barramento cc, onde CH.1 indica se $v_{1}$ é maior do que $v_{2}$ (nível alto) ou vice-versa. CH.3 e 4 apresentam as tensões de saída $v_{1}$ e $v_{2}$, com $5 \mathrm{~V} /$ div.

\subsection{Modelo Discreto LTI para a Malha de Corrente}

Uma malha de corrente com larga banda passante deve ser projetada para fazer a corrente no indutor seguir a corrente referência, em fase com a tensão de entrada retificada $v_{i n}$ tão próxima quanto possível. Para este propósito, um modelo para a corrente no indutor deve ser obtido. Um modelo discreto para grandes sinais pode ser facilmente obtido fazendo-se as seguintes considerações:

(i) A freqüência de comutação é muito maior do que a freqüência da rede, de forma que a tensão de entrada pode ser considerada constante entre dois períodos de comutação;

(ii) A lógica de chaveamento que garante a divisão simétrica de tensão do barramento cc está operando corretamente, de forma que $v_{1}=v_{2}$;

(iii) As duas tensões de saída são constantes dentro de dois períodos de comutação;

(iv) O conversor opera em CCM. 
Desta forma, os possíveis modos de operação do retificador TLB-PFC podem ser representados pela Fig. 4.

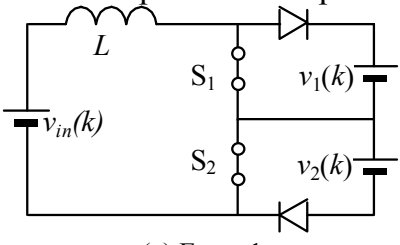

(a) Etapa 1

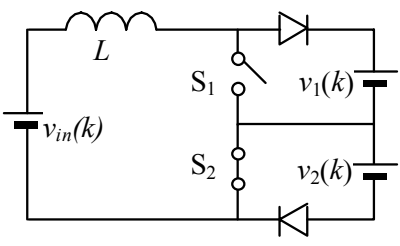

(c) Etapa 3

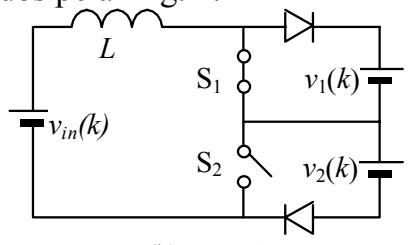

(b) Etapa 2

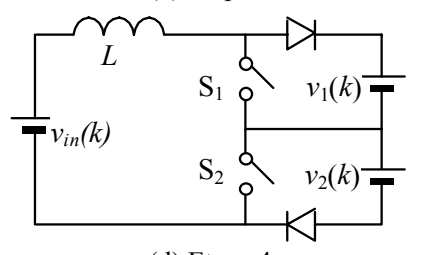

(d) Etapa 4
Fig. 4 - Representação simplificada do retificador TLB-PFC.

Devido ao uso da lógica de chaveamento para a divisão simétrica de tensão, pode-se concluir que na região 1 ocorrem as etapas 1,2 e 3 , enquanto que na região 2 ocorrem as etapas 2, 3 e 4 . Além disto, sendo que $v_{1}$ e $v_{2}$ são iguais, o comportamento da corrente no indutor é o mesmo durante as etapas 2 e 3. Desta forma, resolvendo-se a equação diferencial que descreve o comportamento da corrente no indutor em CCM desde o início até o fim de um período de amostragem (comutação), o modelo discreto linear variante no tempo pode ser obtido (1).

$i_{L}(k+1)=\left\{\begin{array}{l}i_{L}(k)+\frac{v_{\text {in }}(k) T}{L}-\frac{v_{o}(k) T}{2 L}+\frac{v_{o}(k) u(k) T}{2 L}, \text { na região 1 } \\ i_{L}(k)+\frac{v_{\text {in }}(k) T}{L}-\frac{v_{o}(k) T}{L}+\frac{v_{o}(k) u(k) T}{2 L}, \text { na região 2 }\end{array}\right.$

onde $T$ é o período de amostragem, que neste caso é o mesmo período de comutação.

Definindo-se $u_{L}(k)$ conforme (2)

$u_{L}(k)=\left\{\begin{array}{l}\frac{v_{i n}(k) T}{L}-\frac{v_{o}(k) T}{2 L}+\frac{v_{o}(k) u(k) T}{2 L}, \text { na região } 1 \\ \frac{v_{i n}(k) T}{L}-\frac{v_{o}(k) T}{L}+\frac{v_{o}(k) u(k) T}{2 L}, \text { na região } 2\end{array}\right.$

o seguinte modelo LTI é obtido:

$$
i_{L}(k+1)=i_{L}(k)+u_{L}(k) \text {. }
$$

Como (3) representa um modelo discreto LTI, um controlador discreto linear pode ser projetado para produzir $u_{L}(k)$. Deve-se salientar que $u_{L}(k)$ deve ser convertido para $u(k)$ a cada período de amostragem. Isto é realizado utilizando-se (4).

$$
u(k)= \begin{cases}\left(\frac{u_{L}(k) L-v_{\text {in }}(k) T}{v_{o}(k) T}\right) 2+1, & \text { na região } 1 \\ \left(\frac{u_{L}(k) L-v_{\text {in }}(k) T}{v_{o}(k) T}+1\right) 2, & \text { na região } 2\end{cases}
$$

\subsection{Modelo Discreto LTI para a Malha de Tensão}

O uso de modelos linearizados para sistemas não lineares é geralmente adotado para o projeto de controladores para a malha de tensão de conversores boost $[6,11,12]$. Para reduzir os problemas com modelos médios, um modelo linear de grandes sinais para o conversor boost com malha interna de corrente foi apresentado em [11], onde o quadrado da tensão de saída é utilizado como variável de estado. Este método possibilita a obtenção de um modelo simples de primeira ordem.

Para se obter um modelo discreto para o projeto do controlador da malha externa de tensão, assume-se que a corrente no indutor segue a referência com erro nulo. Pelo princípio da conservação de energia, pode-se obter (5).

$$
e_{\text {in }}=e_{L}+e_{C_{c c}}+e_{\text {out }}
$$

onde $e_{i n}, e_{L}, e_{C_{c c}}$ e $e_{o u t}$ são respectivamente a energia fornecida pela rede, a energia armazenada no indutor, a energia armazenada nos capacitores e a energia entregue à carga.

Derivando-se ambos os lados de (5), obtém-se:

$$
\frac{d\left(e_{\text {in }}\right)}{d t}=\frac{d\left(e_{L}\right)}{d t}+\frac{d\left(e_{C_{c c}}\right)}{d t}+\frac{d\left(e_{\text {out }}\right)}{d t}
$$

ou

$$
p_{\text {in }}=\frac{d}{d t} e_{L}+\frac{d}{d t} e_{C_{c c}}+p_{\text {out }}
$$

onde $p_{\text {in }}$ e $p_{\text {out }}$ são respectivamente a potência de entrada e a potência de saída do TLB-PFC.

Substituindo-se em (7) as equações da energia armazenada no indutor e a energia armazenada nos capacitores, obtém-se:

$$
p_{\text {in }}=\frac{1}{2} L \frac{d}{d t} i_{L}^{2}+\frac{1}{2} C \frac{d}{d t} v_{o}^{2}+p_{\text {out }}
$$

onde

$$
C=\frac{C_{1}+C_{2}}{2}
$$

Eq. (8) pode ser simplificada, considerando-se que a energia armazenada no indutor é muito menor do que a energia armazenada nos capacitores de saída [16], portanto,

$$
v_{\text {in }} i_{L}=\frac{1}{2} C \frac{d}{d t} v_{o}^{2}+p_{\text {out }} \text {. }
$$

Uma vez que a corrente no indutor está em fase com a tensão de entrada, (10) pode ser reescrita conforme (11).

$$
\begin{aligned}
& v_{\text {in. }}\left(u_{c c} \cdot v_{\text {in }}\right)=\frac{1}{2} C \frac{d}{d t} v_{o}{ }^{2}+p_{\text {out }} \\
& \frac{1}{2} C \frac{d}{d t} v_{o}{ }^{2}=v_{\text {in. }}\left(u_{c c} \cdot v_{\text {in }}\right)-p_{\text {out }}
\end{aligned}
$$

onde $u_{c c}$ é a ação de controle da malha de tensão, sendo considerada constante dentro de meio período da tensão de entrada.

Integrando-se ambos os lados de (12) do início até o fim de meio ciclo da tensão de entrada, obtém-se:

$$
\begin{aligned}
& \int_{k_{v} \mathrm{~T}_{\mathrm{cc}}}^{\left(k_{v}+1\right) \mathrm{T}_{\mathrm{cc}}} \frac{1}{2} C_{c c} \frac{d}{d t} \mathbf{x}=\int_{k_{v} \mathrm{~T}_{\mathrm{cc}}}^{\left(k_{v}+1\right) \mathrm{T}_{\mathrm{cc}}} u_{c c} v_{\mathrm{p}}^{2} \sin ^{2}(\omega t) d t \\
& -\int_{k_{v} \mathrm{~T}_{\mathrm{cc}}}^{\left(k_{v}+1\right) \mathrm{T}_{\mathrm{cc}}} p_{\text {out }} d t
\end{aligned}
$$

onde $x=v_{o}^{2}(t)$ e $v_{\text {in }}^{2}=v_{\mathrm{p}}^{2} \sin ^{2}(\omega t)$.

A solução de (13) é:

$$
x\left(k_{v}+1\right)=x\left(k_{v}\right)+\frac{u_{c c}\left(k_{v}\right) v_{\mathrm{p}}^{2} T_{c c}}{C_{c c}}-\frac{2 P\left(k_{v}\right) T_{\mathrm{cc}}}{C_{c c}}
$$

ou

$$
x\left(k_{v}+1\right)=G_{c c} x\left(k_{v}\right)+H_{c c} u_{c c}\left(k_{v}\right)+F_{c c} P\left(k_{v}\right)
$$


onde

$$
G_{c c}=1 \quad H_{c c}=\frac{T_{c c} v_{\mathrm{p}}^{2}}{C_{c c}} \quad F_{c c}=-\frac{2 T_{c c}}{C_{c c}}
$$

Devido ao uso de $v_{o}^{2}$ como variável de estado ao invés de $v_{o}$, a representação por variáveis de estado (15) é LTI, onde $u_{c c}\left(k_{v}\right)$ é a ação de controle e $P\left(k_{v}\right)$ é um distúrbio proporcional ao valor médio da potência de saída em meiociclo da tensão de entrada.

\section{PROJETO DOS CONTROLADORES}

Nesta seção a malha interna de corrente e a malha externa de tensão serão projetadas de uma forma sistemática usandose o controlador ótimo linear quadrático discreto em regime permanente [15].

\subsection{Controlador da Malha Interna de Corrente}

Um sistema servo com realimentação de estados e controle integral é adotado para controlar a corrente no indutor. O diagrama do controlador é apresentado na Fig. 5. O modelo da planta foi obtido na Seção 2.2 e é representado por (3).

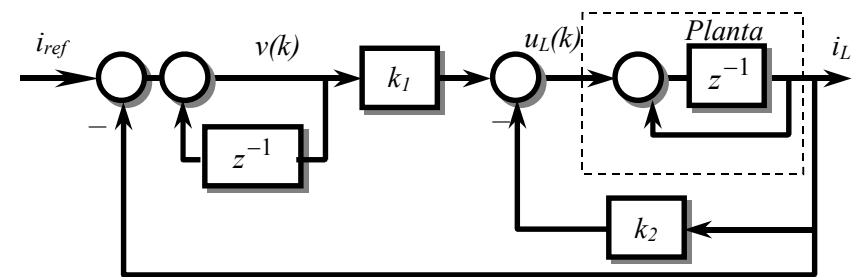

Fig. 5 - Sistema servo com realimentação de estados e ação integral.

As principais equações do sistema são:

$$
\begin{gathered}
i_{L}(k+1)=i_{L}(k)+u_{L}(k) \\
u_{L}(k)=k_{1} v(k)+k_{2} i_{L}(k) \\
v(k)=v(k-1)+i_{\text {ref }}(k)-i_{L}(k)
\end{gathered}
$$

Para a obtenção dos ganhos de realimentação, o projeto do servo de corrente é transformado em um problema de projeto de realimentação de estados. Para este propósito, as variáveis erro de estados são definidas:

$$
\begin{aligned}
& i_{L e}(k)=i_{L}(k)-i_{L}(\infty) \\
& u_{L e}(k)=u_{L}(k)-u_{L}(\infty)
\end{aligned}
$$

Como resultado, a representação por erro de espaço de estados torna-se:

$$
\left[\begin{array}{c}
i_{L e}(k+1) \\
u_{L e}(k+1)
\end{array}\right]=\left[\begin{array}{cc}
1 & 1 \\
0 & 0
\end{array}\right]\left[\begin{array}{c}
i_{L e}(k) \\
u_{L e}(k)
\end{array}\right]+\left[\begin{array}{l}
0 \\
1
\end{array}\right] w(k)
$$

onde

$$
w(k)=\left[\begin{array}{ll}
-k_{1} & 1-k_{2}
\end{array}\right]\left[\begin{array}{c}
i_{L e}(k) \\
u_{L e}(k)
\end{array}\right]
$$

Os ganhos de realimentação $k_{1}$ e $k_{2}$ foram obtidos utilizando-se o a técnica de controle ótimo Regulador Quadrático Linear Digital (digital linear quadratic regulator - DLQR) para garantir a performance desejada em malha fechada [15]. Os ganhos do controlador são selecionados de modo a minimizar a função custo discreta (23). O sistema é assimptoticamente estável se a matriz $\mathbf{Q}_{\mathbf{i}}$ é definida positiva ou Hermitiana semidefinida positiva e $R_{i}$ é positivo. Mesmo que o sistema resultante seja sempre estável, a performance do sistema depende dos valores especificados em $\mathbf{Q}_{\mathbf{i}}$ e $R_{i}$.
Uma vez que $\mathbf{Q}_{\mathrm{i}}$ e $R_{i}$ são definidos, os ganhos $k_{1}$ e $k_{2}$ podem ser obtidos a partir da solução recursiva da equação de Ricatti.

$$
J=\frac{1}{2} \sum_{k=0}^{\infty} \mathbf{x}_{\mathbf{i}}^{\mathbf{T}}(k) \mathbf{Q}_{\mathbf{i}} \mathbf{x}_{\mathbf{i}}(k)+u_{L}(k) R_{i} u_{L}(k)
$$

É importante salientar que o diagrama apresentado na Fig. 5 não considera o tempo requerido para se computar a lei de controle. Este tempo gasto com o processamento produz um atraso que torna-se significativo quando se opera em altas freqüências de amostragem/comutação. Portanto, a compensação deste tempo de atraso é necessária para evitar degradação da performance do controlador $[8,13,14]$. Neste artigo, um observador de estados preditivo é utilizado para estimar a corrente no indutor no próximo período. A corrente estimada no próximo período $\hat{i}_{L}(k+1)$ é então utilizada para a obtenção da ação de controle $u_{L}(k+1)$ para o próximo período.

$$
\begin{gathered}
u_{L}(k+1)=v(k+1) \cdot k_{1}+\hat{i}_{L}(k+1) \cdot k_{2} \\
v(k+1)=v(k)+i_{r e f}(k)-\hat{i}_{L}(k)
\end{gathered}
$$

$\mathrm{O}$ diagrama de blocos do controlador com o observador de estados preditivo é apresentado na Fig. 6 .

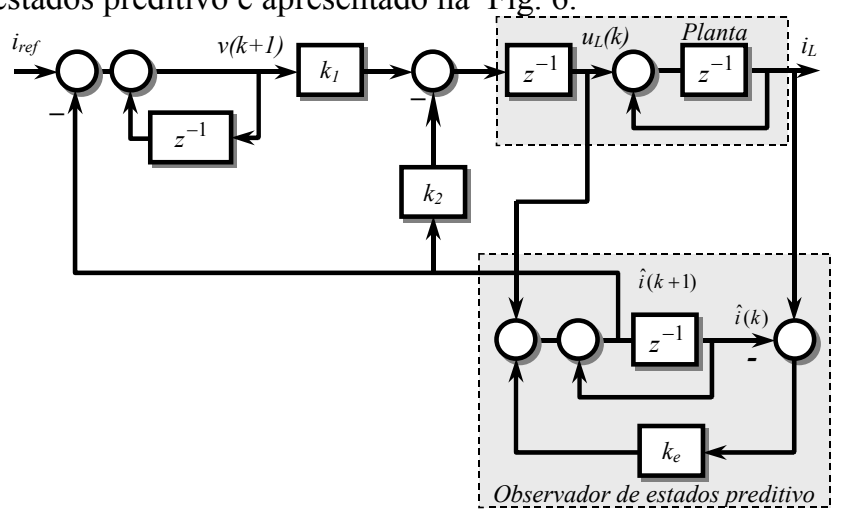

Fig. 6 - Sistema servo com realimentação de estado observado e controlador integral.

A corrente estimada $\hat{i}_{L}(k+1)$ é dada por:

$$
\hat{i}_{L}(k+1)=\left(1-k_{e}\right) \hat{i}_{L}(k)+u_{L}(k)+k_{e} \cdot i_{L}(k)
$$

O observador de estados (10) é assimptoticamente estável se $0<k_{e}<1$, onde o ganho do observador $k_{e}$ pode ser definido de diferente formas: (i) utilizando-se a técnica do filtro de Kalmann; (ii) fazendo-se $k_{e}=1$, resultando em um observador com resposta dead-beat; (iii) heuristicamente, selecionandose um valor entre zero e um para rejeitar ruídos de aquisição presentes nos sistema.

A Fig. 7 representa o diagrama de blocos completo do controlador da malha interna de corrente proposto para $\mathrm{o}$ retificador TLB-PFC.

Os ganhos de realimentação utilizados são $k_{1}=0.5$ e $k_{2}=1$, sendo que as matrizes $\mathbf{Q}_{\mathrm{i}}$ e $R_{i}$ utilizadas foram $\mathbf{Q}_{\mathrm{i}}=\operatorname{diag}(1,1)$ e $R_{i}=1$. A Fig. 8 apresenta a resposta transiente simulada e experimental devido a um degrau na corrente de referência $i_{\text {ref. }}$ A comparação dos resultados valida o projeto desenvolvido, onde pode-se perceber a conformidade dos resultados experimentais com o resultados obtidos através de um processo interativo. 


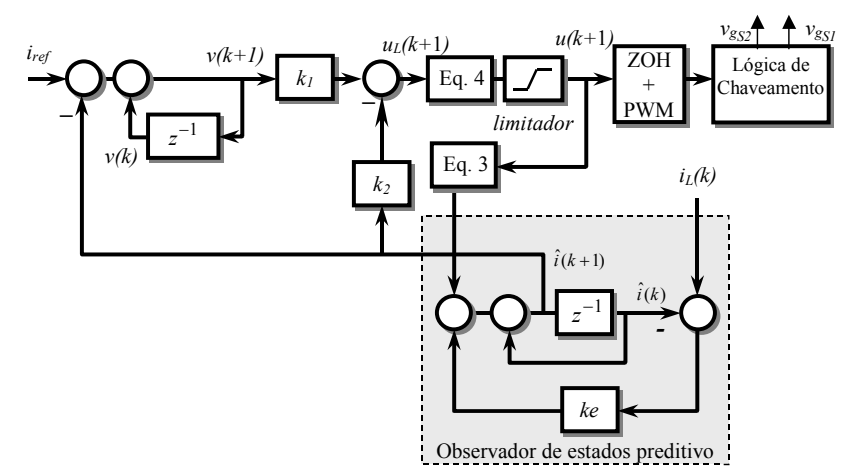

Fig. 7 - Controlador da malha interna de corrente proposto para o retificador TLB-PFC.

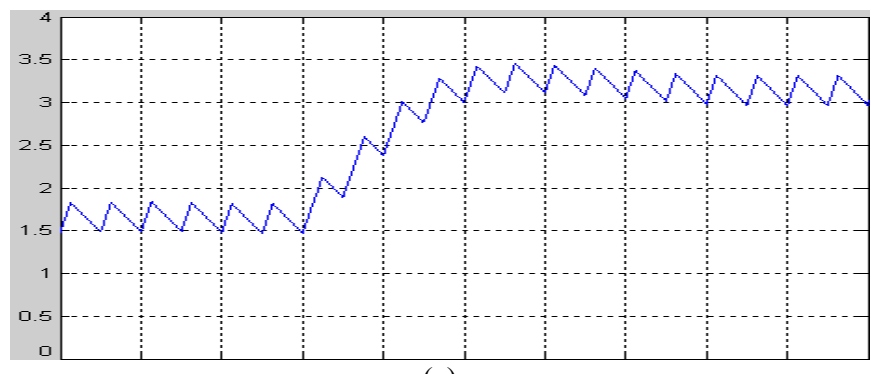

(a)

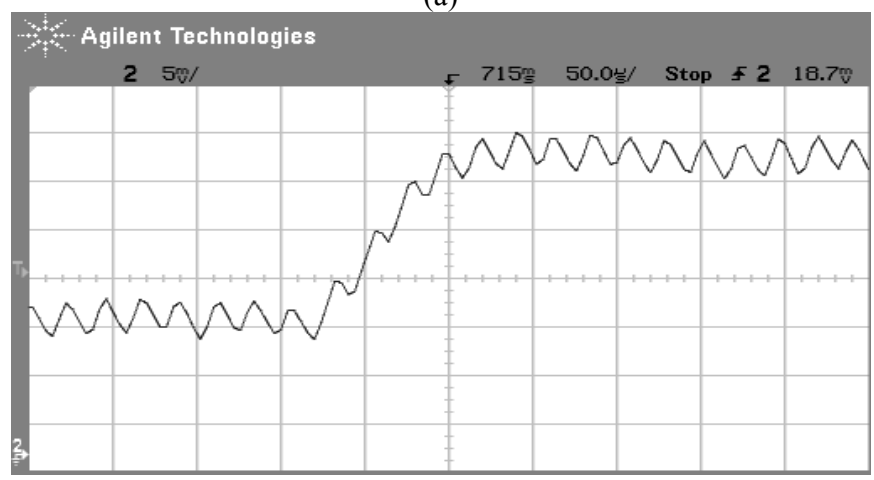

(b)

Fig. 8 - Corrente no indutor para um degrau em $i_{\text {ref: }}$ (a) Resultado de simulação; (b) Resultado experimental (500mA/div.).

\subsection{Controlador da Malha Externa de Tensão}

De um modo similar à malha interna de corrente, um sistema servo com realimentação de estados e controle integral é utilizado para o controle da tensão do barramento cc. O diagrama de blocos do controlador é apresentado na Fig. 9, sendo que o modelo da planta foi obtido na Seção 3.1 e é representado por (15).

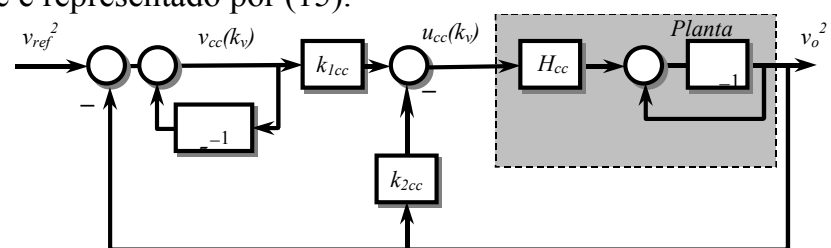

Fig. 9 - Sistema servo com realimentação de estados para a malha externa de tensão.

A partir da Fig. 9 a ação de controle $u_{c c}(k)$ e a variável erro integrado $v_{c c}(k)$ podem ser obtidos:

$$
\begin{gathered}
u_{c c}\left(k_{v}\right)=k_{1 \mathrm{cc}} v_{c c}\left(k_{v}\right)-k_{2 c c} x\left(k_{v}\right) \\
v_{c c}\left(k_{v}\right)=v_{c c}\left(k_{v}-1\right)+v_{r e f}^{2}\left(k_{v}\right)-v_{o}^{2}\left(k_{v}\right)
\end{gathered}
$$

Os ganhos de realimentação do controlador $k_{1 c c}$ e $k_{2 c c}$ podem ser obtidos utilizando-se os mesmos procedimentos descritos na Seção 3.1.

Embora controladores rápidos tenham sido apresentados $[3,4,6,11]$, aqui a freqüência de amostragem da malha externa de tensão é mantida em $120 \mathrm{~Hz}$ para reduzir os esforços computacionais bem como para melhorar o fator de potência da entrada. Os ganhos de realimentação utilizados para a malha externa de tensão foram $k_{1 c c}=1.7244$ e $k_{2 c c}=4.2859$, os quais foram obtidos utilizando-se $\mathrm{Q}=\operatorname{diag}(10,2)$ e $\mathrm{R}=0.01$.

\section{RESULTADOS EXPERIMENTAIS}

Um protótipo de $1.5 \mathrm{kVA}$ operando a $45 \mathrm{kHz}$ foi implementado para demonstrar o comportamento dos controladores e modelos discretos propostos. As leis de controle foram implementadas em um único processador de sinais digitais (digital signal processor - DSP) de ponto fixo TMS320F241, o qual apresenta internamente 3 geradores de PWM. O período de amostragem para a malha de corrente é $\mathrm{T}=22,22 \mu \mathrm{s}(45 \mathrm{kHz})$, enquanto que a malha de tensão é efetuada somente uma vez a cada $8,33 \mathrm{~ms}(120 \mathrm{~Hz})$, quando a tensão de entrada cruza por zero. Salienta-se que quando a malha de tensão é efetuada, a malha de corrente não é calculada. Este procedimento não produz distorção de corrente, uma vez que isto ocorre quando a tensão de entrada é próxima de zero e a variável de controle $u$ praticamente não muda nesta região [2]. A Fig. 10 demonstra como as rotinas de controle são executadas dentro de um semi-ciclo de rede.

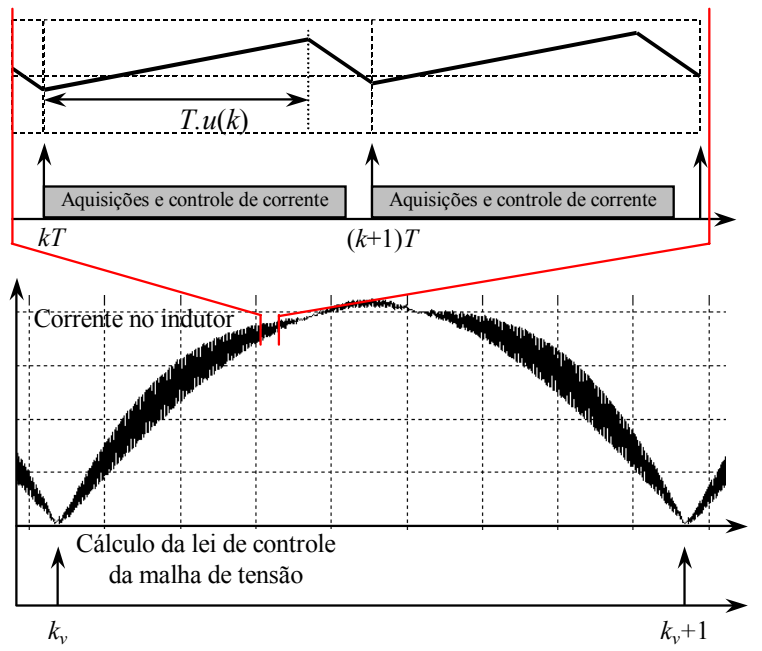

Fig. 10 - Distribuição das rotinas de controle em meio-ciclo de rede.

A relação de componentes utilizados no protótipo está apresentada na TABELA II.

TABELA II

Lista de Componentes e Dispositivos Utilizados no Protótipo.

\begin{tabular}{ll}
\hline \multicolumn{1}{c}{ Componente } & \multicolumn{1}{c}{ Descrição } \\
\hline $\mathrm{S}_{1}, \mathrm{~S}_{2}$ & IGBTs 27N60C3DR \\
$\mathrm{D}_{1}, \mathrm{D}_{2}$ & HFA25PB60 \\
$L$ & $1 \mathrm{mH}$ \\
$C_{1}, C_{2}$ & $470 \mu \mathrm{F} / 400 \mathrm{~V}$ \\
\hline
\end{tabular}

A Fig. 11 apresenta a tensão de entrada e a corrente de entrada, para operação a plena carga. O fator de potência da corrente de entrada é muito próximo de um $(\mathrm{PF}=0.995)$. 


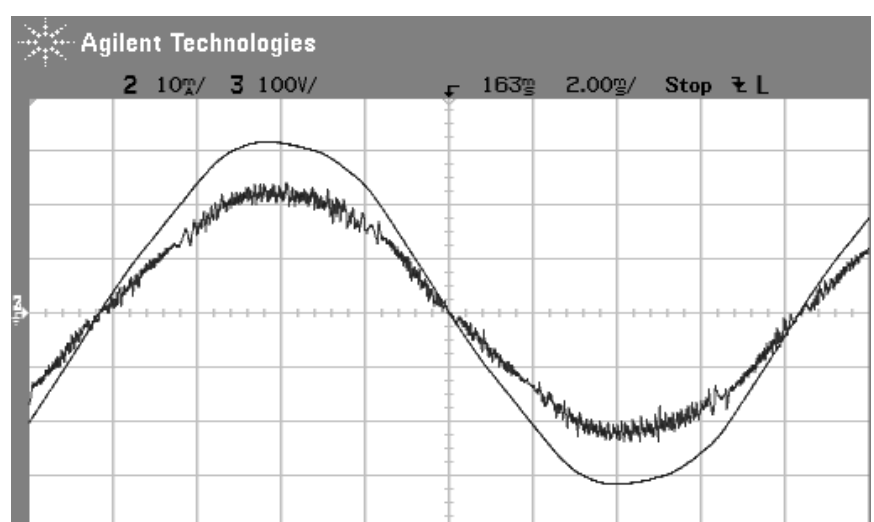

Fig. 11 - Resultados experimentais: corrente de entrada (5A/div) e tensão de entrada $v_{i n}(100 \mathrm{~V} /$ div $)$.

A Fig. 12 apresenta resultados de simulação e resultados experimentais para um degrau de $50 \%$ a $100 \%$ de plena carga. Pode-se verificar a boa performance do sistema para o transiente, bem como a validação da análise desenvolvida, uma vez que os resultados experimentais apresentam boa correspondência com os resultados de simulação.

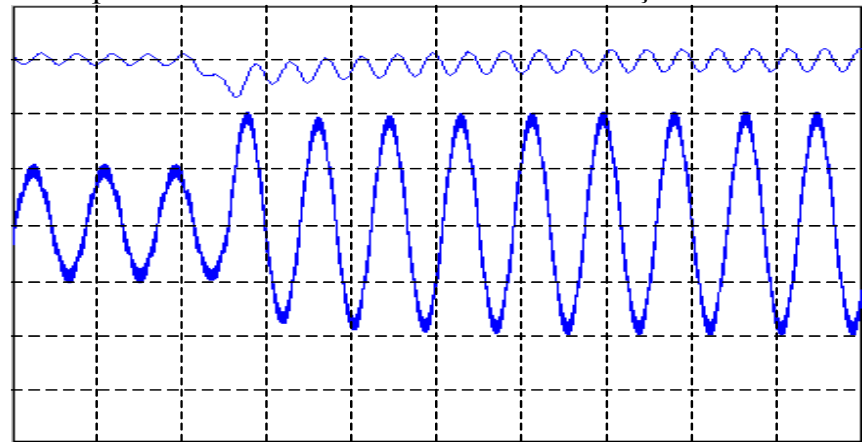

(a)

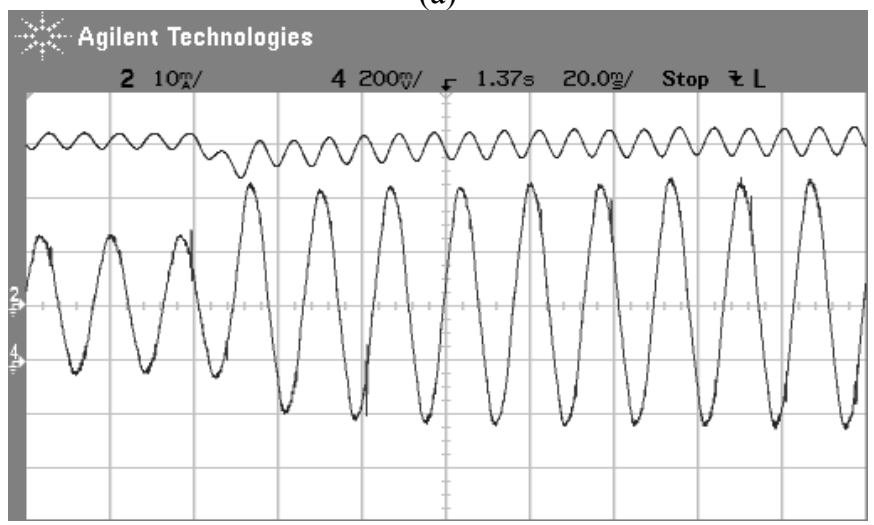

(b)

Fig. 12 - Corrente no indutor e tensão de saída $v_{o}$ para um degrau de carga de 50\% a 100\% no retificador TLB-PFC: (a) Resultados de simulação; (b) Resultados experimentais.

A Fig. 13 apresenta o conteúdo harmônio requerido pela norma (IEC61000-3-2) e o conteúdo harmônico para a corrente de entrada para as harmônicas pares e ímpares. Pode-se perceber que o conteúdo harmônico do retificador TLB é menor do que os limites especificados pelas normas para equipamentos da classe A. Harmônicas de $9^{\text {a }}$ ordem em diante não são apresentadas neste gráfico, já que elas são muito menores e estão em conformidade com as exigências da norma.

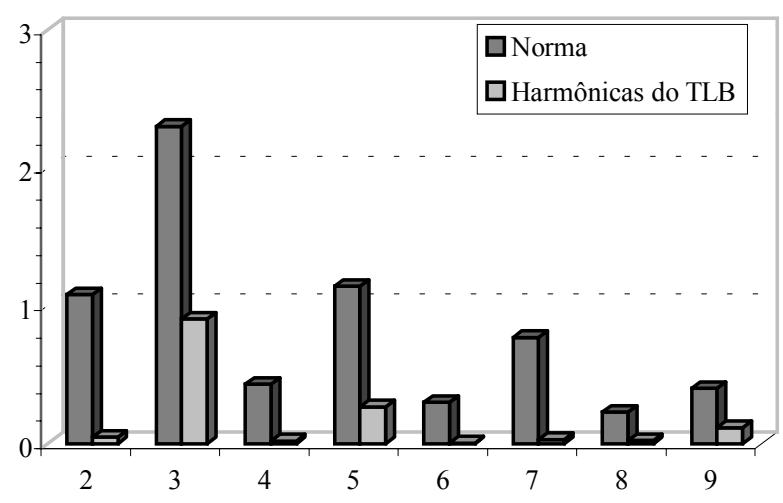

Fig. 13 - Limites de harmônicas da norma e harmônicas do TLB.

\section{CONCLUSÃO}

Este artigo apresenta controladores discretos para o retificador com fator de potência corrigido boost três níveis. Modelos discretos lineares para ambas as malhas de tensão e corrente são apresentadas e validadas experimentalmente.

Controladores servos com realimentação de estados são utilizados em ambas as malhas de tensão e de corrente. Um procedimento sistemático para selecionar os ganhos de realimentação é apresentado baseado no regulador quadrático linear discreto, o qual garante comportamento assimptoticamente estável para a malha de corrente e a malha de tensão.

Para compensar atrasos devido à computação das leis de controle, um observador de estados preditivo foi projetado e implementado. Além de compensar o tempo de atraso, o observador fornece um grau adicional de liberdade para a filtragem dos ruídos presentes na amostragem da corrente no indutor.

Resultados experimentais de um protótipo de $1,5 \mathrm{~kW}$ validam a análise desenvolvida, bem como demonstram a boa performance do retificador TLB-PFC com os controladores discretos propostos.

\section{AGRADECIMENTOS}

Os autores agradecem à Thornton Inpec Eletrônica LTDA e à Epcos capacitores pela doação de componentes utilizados neste trabalho, e à CAPES pelo apoio financeiro.

\section{REFERÊNCIAS BIBLIOGRÁFICAS}

[1] M.T. Zhang, Y.Jing, F.C. Lee, M.Jovanovic, "Singlephase three-level boost power factor correction converter", Applyed Power Electronics and Specialists Conference, pp. 434-439, 1995.

[2] J.R. Pinheiro, D.L.R. Vidor, H.A. Gründling, "Dual output three-level boost power factor correction converter with unbalanced loads", Power Electronics Specialists Conference, vol. 1, pp. 733-737, 1996.

[3] G. Spiazzi, P. Mattavelli, L. Rossetto, "Power factor preregulators with improved dynamic response", IEEE Trans. on Power Electronics, vol. 12, no. 2, pp. 343349, 1997. 
[4] M.O. Eissa, S.B. Leeb, G.C. Verghese, A.M. Stankovic, "Fast controller for a unity-power-factor PM rectifier", IEEE Trans. on Power Electronics, vol. 11, no. 1, pp. 16, 1996.

[5] A.S. Martins, E.V. Kassick, I. Barbi, "Control strategy for the double-boost converter in continuous conduction mode applied to power factor correction", Power Electronics Specialists Conference, vol. 2, pp. 10661072, 1996.

[6] A. Prodic, J. Chen, R.W. Erickson, D. Maksimovic, "Digitally controlled low-harmonic rectifier having fast dynamic responses", Applied Power Electronics Conference, vol. 1, pp. 476-482, 2002.

[7] K. Mahabir, G. Verghese, J. Thottuvelil, A. Heyman, "Linear averaged and sampled data models for large signal control of high power factor ac-dc converters", Power Electronics and Specialists Conference, v.1, pp.372-381, 1990.

[8] S. Bibian, H. Jin, "High performance predictive dead-beat digital controller for dc power supplies", Applied Power Electronics Conference, vol. 1, pp. 67-73, 2001.

[9] C.T. Chen, "Analog \& digital control system design", Saunders College Publishing, 1993.

[10] OGATA K., "Discrete-Time Control Systems", Prentice-Hall, 1987;

[11] A.H. Mitwalli, S.B. Leeb, G.C. Verghese, V.J. Thottuvelil, "An adaptive digital controller for a unity power factor converter", IEEE Trans. on Power Electronics, vol. 11, no. 2, pp. 374-382, 1996.

[12] J.B. William, "Design of feedback loop in unity power factor ac to dc converter", Power Electronics and Specialists Conference, v. 2, pp. 959-967, 1989.

[13] S. Bibian, H. Jin, "A simple prediction technique for the compensation of digital control time delay in $\mathrm{dc}$ switchmode power supplies", Applied Power Electronics Conference, vol. 2, pp. 994-1100, 1999.

[14] C. Rech, H. Pinheiro, H.L. Hey, H.A. Gründling, J.R. Pinheiro "Improved modified OSAP controller for voltage source PWM inverters", Congreso Brasileiro de Eletrônica de Potência, pp. 329-334, 2001.

[15] F. Botterón, H. Pinheiro, H.A. Gründling, J.R. Pinheiro, H.L. Hey, "Digital voltage and current controllers for three-phase PWM inverter for UPS applications", IEEE Ind. Appl. annual Meeting, v. 4, pp. 2667-2674, 2001.

[16] M.O. Eissa, S.B. Leeb, G.C. Verghese, A.M. Stankovic, "Fast controller for a unity-power-factor PWM rectifier", IEEE Trans. on Power Electronics, vol.11, no.1, January 1996.

\section{DADOS BIOGRÁFICOS}

Hélio Leães Hey, nasceu em Santa Maria - Rio Grande do Sul, em 29 de Julho de 1961. Formou-se em Eng. Elétrica pela Universidade Católica de Pelotas, Pelotas - RS, em 1985. Obteve os títulos de Mestre e Doutor em Eng. Elétrica pela Universidade Federal de Santa Catarina, Florianópolis SC, em 1987 e 1991 respectivamente. Entre 1989 e 1993, atuou como professor adjunto na Universidade Federal de Uberlândia, Uberlândia-MG. Atualmente, é professor titular do Depto. de Eletrônica e Computação da Universidade
Federal de Santa Maria, Santa Maria-RS. De 1997 à 2000 foi coordenador do programa de pós-graduação em Engenharia Elétrica da UFSM. De 1995 a 1999 foi o editor da Revista Brasileira de Eletrônica de Potência, vinculada a Sociedade Brasileira de Eletrônica de Potência.

Hilton Abílio Gründling nasceu em Santa Maria, RS, Brasil, em 1954. Ele formou-se em engenharia elétrica pela Pontifícia Universidade Católica do Rio Grande do Sul, Porto Alegre, Brasil, em 1977. Ele recebeu o grau de Mestre pela Universidade Federal de Santa Catarina, Santa Catarina, Brasil, em 1980 e o grau de Doutor do Instituto Tecnológico da Aeronáutica, São Paulo, Brasil, em 1995. Desde 1980, tem atuado como professor na Universidade Federal de Santa Maria. Suas áreas de interesse incluem Controle Adaptativo Robusto por Modelo de Referência, Controle Discreto e Aplicações de Sistemas de Controle.

Humberto Pinheiro nasceu em Santa Maria, Brasil, em 1960. Ele recebeu o grau de engenheiro eletricista pela Universidade Federal de Santa Maria, em 1983, o grau de Mestre pela Universidade Federal de Santa Catarina, em 1987, e o grau de doutor pela Concordia Univeristy, Montreal, Canada, em 1999. Ele trabalhou com engenheiro na BK Controles Eletrônicos, de 1983 até 1990 e ministrou eletrônica de potência na PUC-RS de 1987 até 1991. Ele atualmente é Professor Adjunto no Departamento de Eletrônica e Computação na Universidade Federal de Santa Maria, atuando do GEPOC. Entre seus assuntos de pesquisa de interesse estão fontes ininterruptas de energia e controle discreto de conversores estáticos.

José Eduardo Baggio nasceu em Santa Maria - RS, em 1971. Formou-se em Engenharia Elétrica pela Universidade Federal de Santa Maria, em 1995. Obteve o título de Mestre em Engenharia Elétrica na Universidade Federal de Santa Maria, em 1997. Atualmente é estudante de doutorado na mesma universidade. Sua área de interesse compreende Eletrônica de Potência, Sistemas de Controle e Sistemas Digitais.

José Renes Pinheiro nasceu em Santa Maria, RS, Brasil, em 1958. Recebeu o grau de Engenheiro Eletricista pela Universidade Federal de Santa Maria, Santa Maria, Brasil, e os graus de Mestre e Doutor em Engenharia Elétrica pela Universidade Federal de Santa Catarina, Florianópolis, SC, Brasil, em 1981, 1984, e 1994, respectivamente. Atualmente, O Dr. Pinheiro é Professor Titular do Departamento de Eletrônica e Computação da Universidade Federal de Santa Maria, onde atua desde 1985. Em 1987, foi um dos fundadores do Grupo de Eletrônica de Potência e Controle (GEPOC). Foi o coordenador de Programa Técnico do Congresso Brasileiro de Eletrônica de Potência (COBEP), em 1999, e do Seminário de Eletrônica de Potência e Controle (SEPOC), em 2000. Em 2001 e 2002, ele realizou Pós-doutorado na área de Sistemas de Energia Distribuídos, no Center for Power Electronics Systems (CPES), da Virginia Polytechnic Institute and State University (Virginia Tech), Blacksburg, USA. Suas principais linhas de pesquisas 
incluem Sistemas Híbridos de conversão estática de energia, Sistemas de alimentação de alta freqüência, Técnicas de compensação e correção do fator de potência, modelagem e controle de conversores estáticos. Dr. Pinheiro é membro da Sociedade Brasileira de Eletrônica de Potência, da Sociedade Brasileira de Automática, e de Sociedades da IEEE. 